\title{
Establishing an Integrated Quality Assurance and Patient's Safety Service in a Tertially, Academic Medical Center
}

Yaron Niv*, Evegenee Berkov, Nechama Chorev, Sigal Cohen, Pazit Kanter, Uri Gabai, Sharoni Elias, Oshrat Shachak, Yifat Leviron, Tali Weiler, Meital Tobi and Arian Ganor

Department of Quality Assurance and Risk Management, Rabin Medical Center, Clalit Health Services, Tel Aviv University, Israel

\begin{abstract}
Background: Clalit Health Services (CHS) is a large health care provider for 4.6 million enrollees in Israel. This is the country's largest medical organization with an annual budget of approximately 17 billion New Israeli Shekel (NIS) Rabin Medical Center comprising Beilinson and HaSharon hospitals is the biggest medical center of $\mathrm{CHS}$, an academic, tertially, referral center, with 1200 beds, including all services of a modern hospital.
\end{abstract}

Aim: To describe the establishment process of integrated quality assurance and patient's safety service and to evaluate its success in 4 years of activity. Quality indicators (process and outcome) were developed and monitored. Improvement was assessed by comparing the results of 2013 and 2016 indicators.

Methods: We believe in "No blame or shame" and "To err is human" strategy. The patient is always in the center continuous learning is being conducted with conclusions and improvement plans, implementation and systematic approach, measuring and proactive activity to improve patient safety. We used Plan Do CheckAct (PDCA) cycle in most of the processes.

Results: We established 4 units: quality assurance, risk management, regulation committee for policy, strategic affairs and legal aid, and unit of quality indicators and quality working plans. Quality improvement plan was performed every year. We demonstrated a significant improvement in most of the quality indicators measured.

Conclusion: Organizational changes focused on patient safety, based on clinical protocols, quality indicators and special committees, brought the hospital to new, high level, achievements. We believe that our patients enjoy high level quality of care in the hospital safe environment.

Keywords: Quality; Safety; Risk management; Hospital management

\section{Introduction}

Clalit Health Services (CHS) is a large health care provider for 4.6 million enrollees in Israel. This is the country's largest health medical organization (HMO) with an annual budget of approximately 17 billion NIS. The organization has two major medical branches: a community based service divided into eight geographical districts, each with a separate budget and regional director and a hospital division comprising 14 hospitals providing acute care, chronic care and psychiatric care. The administrative structure has undergone a series of changes in the last three decades to improve the function of this large organization. Under this system each community-based regional branch of CHS purchases essential services from hospitals. Rabin Medical Center (RMC) comprising Beilinson and HaSharon hospitals is the biggest medical center of CHS, an academic, tertially referral center, with 1200 beds, including all services of a modern hospital. In 2012 it was decided to establish an integrated quality assurance and patient's safety central service (QSS) for Rabin Medical Center, to provide a comprehensive service to the hospital departments, advancing quality and safety of patients' management. In this manuscript we describe the establishment process of the QSS and evaluate its success in 4 years of activity. Quality indicators (process and outcome) were developed and monitored. Improvement was assessed by comparing 2013 to 2016 indicators.

\section{Vision}

The vision of the new QSS was to provide the best treatment for every patient, with maximal safety and efficiency, in a reasonable cost. Our quality plan includes advancing treatment quality, supporting the patient and his/her family, leadership in medicine, medical sciences and education, improving screening outcomes, and making safety and quality the interest of all personnel.
We believe in "No blame or shame" and "To err is human" strategy [1]. The patient is always in the center, regular and continuous learning are being conducted with conclusions and improvement plans, implementation and systematic approach, measuring and proactive activity to improve patient safety. We used Plan Do Check Act (PDCA) cycle in most of the processes (Figure 1).

\section{Integrated quality assurance and patient's safety service (QSS) - structure}

On 01.07.12 the QSS was officially established, together with a number of organizational changes. The QSS is the product of cooperation between RMC and the general management of CHS. The project was encouraged by CHS's director and deputy director of the hospitals division. The structure of QSS is demonstrated in Figure 2. We established 4 different units: quality assurance unit, risk management unit, regulation committee for policy, strategic affairs and legal aid and unit of quality indicators and quality working plans. We also nominated members of the QSS for all the

*Corresponding author: Yaron Niv, Department of Quality Assurance and Risk Management, Rabin Medical Center, Clalit Health Services, Tel Aviv University, Israel Tel: 03-9377329; Email: yniv@Clalit.org.il, nivyaron80@gmail.com

Received May 11, 2017; Accepted June 03, 2017; Published June 10, 2017

Citation: Niv Y, Berkov E, Chorev N, Cohen S, Kanter P, et al. (2017) Establishing an Integrated Quality Assurance and Patient's Safety Service in a Tertially, Academic Medical Center. Health Care Current Reviews 5: 198. doi: 10.4172/23754273.1000198

Copyright: @ 2017 Niv Y, et al. This is an open-access article distributed unde the terms of the Creative Commons Attribution License, which permits unrestricted use, distribution, and reproduction in any medium, provided the original author and source are credited. 
Citation: Niv Y, Berkov E, Chorev N, Cohen S, Kanter P, et al. (2017) Establishing an Integrated Quality Assurance and Patient's Safety Service in a Tertially, Academic Medical Center. Health Care Current Reviews 5: 198. doi: 10.4172/2375-4273.1000198

PDCA includes the following steps:
- Plan the improvement and continued data collection
- Do improvement, data collection and analysis
- Check the results and determine if the actions were effective
Act to spread improvements and maintain them

Figure 1: PDCA (Plan Do Check Act) cycle. medical center institutional committees such as drug committee, resuscitation committee, etc.

\section{QSS Functions and Activity}

The functions of QSS and specific units are described in Figure 3. RMC provides a systematic approach to the maintenance and improvement of patient safety, effective responses to actual occurrences, on-going proactive reduction in medical care errors and integration of patient safety priorities into all relevant processes, functions and services. The specific activity of the year 2016 is shown in Figure 4.

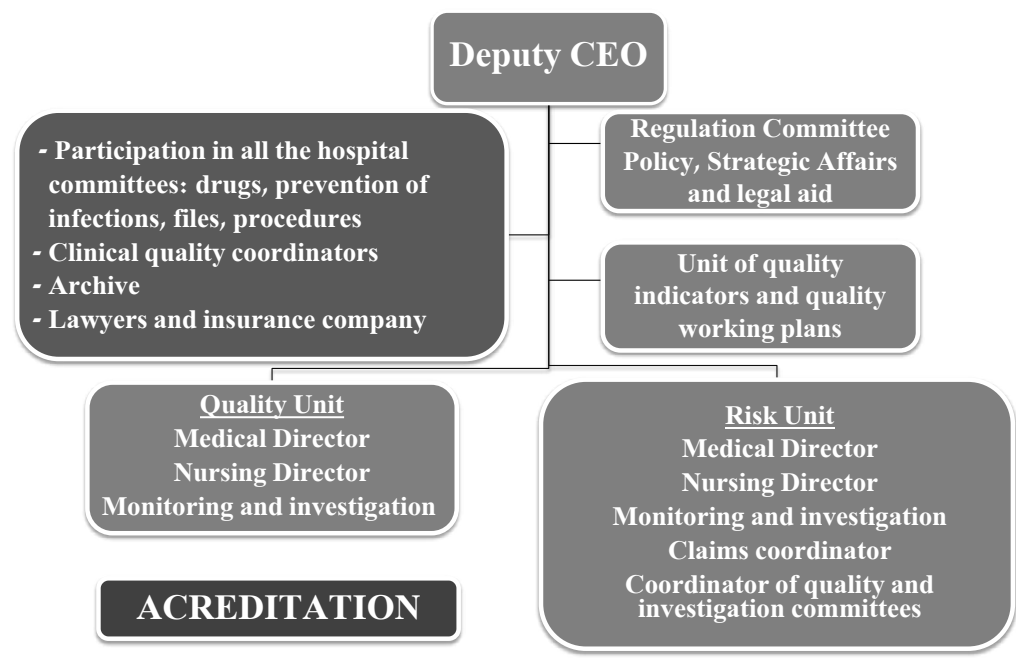

Figure 2: Structure of the department of quality assurance and risk management.
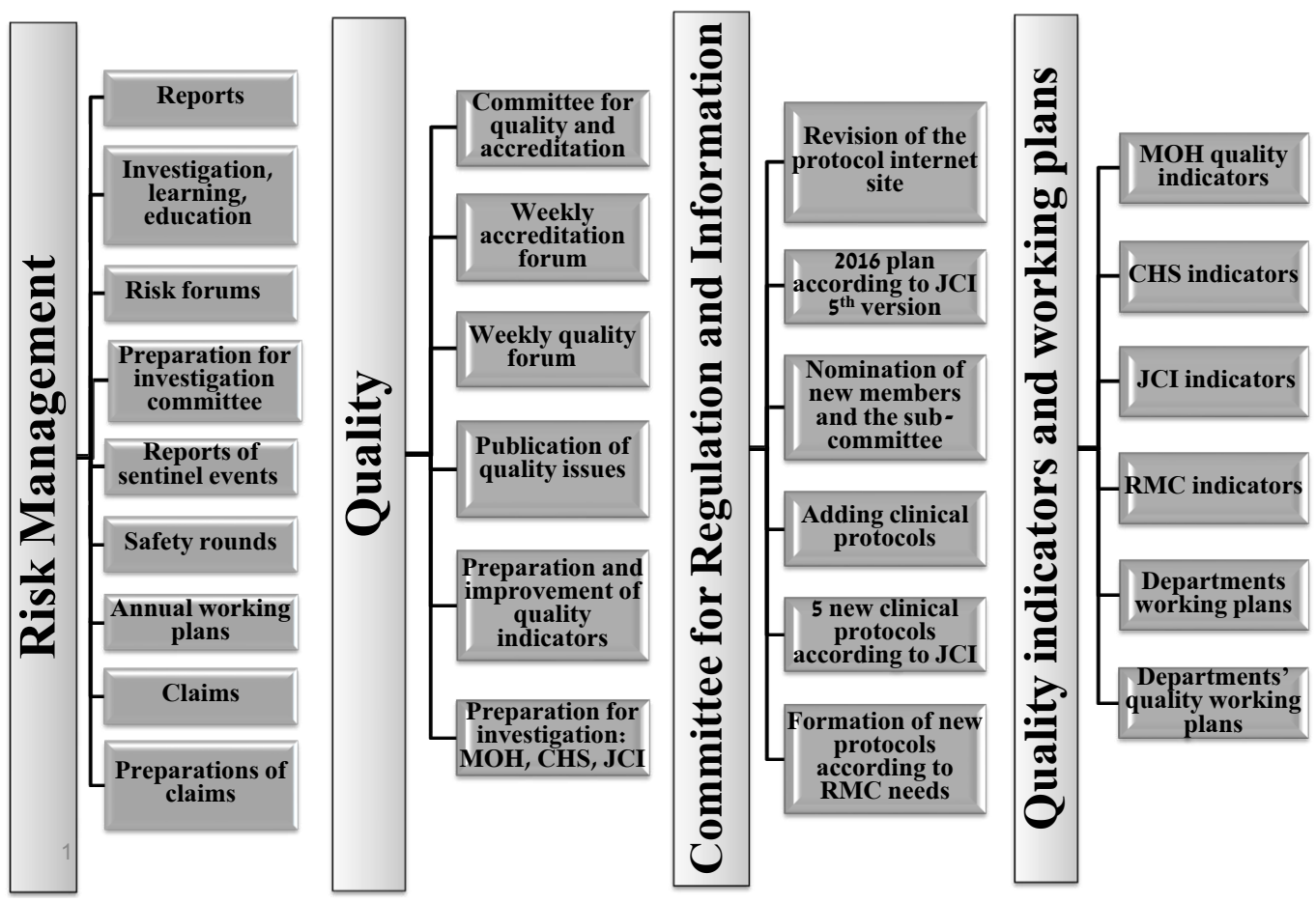

Figure 3: The functions of QSS and the specific units. 


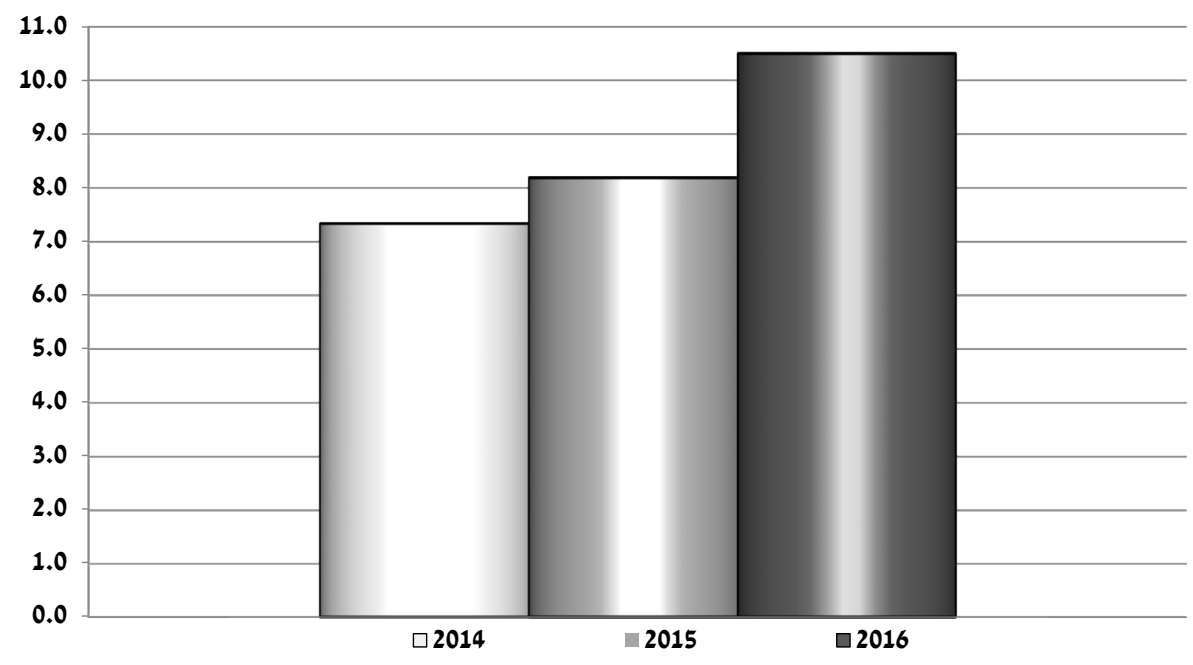

Figure 4: Near miss reports 2016.

\section{Proactive Activity, CHS Working Plan Milestones}

Proactive activity to improve patients' safety was taken according to CHS working plan and RMC policy: Safety rounds in all the departments guided by the hospital director and his deputies, organizational learning from adverse events, encouraging nearmiss reports, encouraging patients to become active members of their health care team, proactive activities collaborating with the pharmacy, improving ISBAR (Introduction, Situation, Background, Assessment, Recommendation) communication, performing FMEA (Failure Mode and Effects Analysis) for risk subjects to prevent failures, immediate mediation, participating in quality and risk management courses, performing trigger tool analysis in obstetrics, quality and control committees, investigation committee reports and conclusions, monitoring time out in the operating rooms, readmission to the emergency department, proper patient identification, step out protocol in patients transfer, patient empowerment, diagnosis mistakes and risk management forums.

As example we demonstrated a significant increase in "near miss" reports in 2016 (Figure 5). The main findings in the safety round that direct our attention in 2016 was the knowledge of the staff how to behave in the case of fire and prevention of blockade of important exits.

\section{Reporting Major Events}

Staff members involved in a major event occurrence receives support regarding the professional and emotional reconciliation of the event. Staff involvement in the root cause analysis and action plan processes is encouraged to have an active role in the process resolution. External reporting is performed in accordance with all rules, laws and requirements. Medical care errors and occurrences are reported internally and externally per hospital policy, and through the channels established by this plan. Investigations, root cause analysis, FMEA, Quality Committee or Investigation Committee performed as needed (Figure 4). We had 52 claim meetings in 2016 for preparing and assessing the possibility of defending or compromising cases and 5 meeting of "Claims Mirror" in different disciplines - showing the mistakes done in their particular cases.

\section{Management Priority Issues and Clinical Protocols}

Five priority issues protocols are chosen every year according to the hospital performance (examples: falls, pressure sores, central line sepsis), according to reporting of sentinel events, adverse events, nearmiss events, frequent claims and complains, recommendation of quality committee, or the general quality indicators cited by the ministry of health (MOH), CHS, RMC management and the Joint Commission International (JCI). The clinical protocols deal with issues that are more prevalent or more severe, with high volume, and based upon the computer systems, digital patient files, and analysis performed by the Quality unit. For every clinical protocol we used the PDCA cycle and we have at least one or two indicators (process and outcome). The clinical protocols for 2016 were: communication between staff members using the ISBAR language in critical patient transfer, hand hygiene in 2 specific World Health Organization (WHO) moments - before contact with the patient and before aseptic procedure, prevention of pressure ulcers, encouraging reports: near miss, adverse events and drug sideeffects and education for fire prevention and behavior.

We found that most of the "near miss" reports were due to failure in communication, in patients' transfer, patients' identification and medication. Hand hygiene for more than 400 opportunities inspected improved in all hospital sectors in the 2nd quarter of 2016, being $94 \%$ for ventilation technicians, $88 \%$ for physiotherapists, $83 \%$ for radiology technicians but less than the goal of $70 \%$ for all other sectors. Continuous improvement was achieved for pressure ulcers following intensive campaign and many tracers (Figure 6). In 2016 90\% of the staff had fire guidance, $100 \%$ of the departments had fire evacuation drills and $50 \%$ of nurses and physicians participated in fire simulation drill.

In addition to the "priority issues" 5 clinical protocols were chosen every year. In 2016 we selected percutaneous coronary intervention (PCI) within 90 min of arrival to the emergency department in patients with ST elevation myocardial infarction (STEMI), anti-thrombotic treatment to high-risk patients for deep vein thrombosis (DVT), tissue plasminogen activator (TPA) or performance of neuroangiography to acute cerebrovascular accident (CVA) patients, pain treatment after surgery in the recovery room, and controlling hyperglycemia during hospitalization. Comprehensive protocols for each of these issues were 
Citation: Niv Y, Berkov E, Chorev N, Cohen S, Kanter P, et al. (2017) Establishing an Integrated Quality Assurance and Patient's Safety Service in a Tertially, Academic Medical Center. Health Care Current Reviews 5: 198. doi: 10.4172/2375-4273.1000198

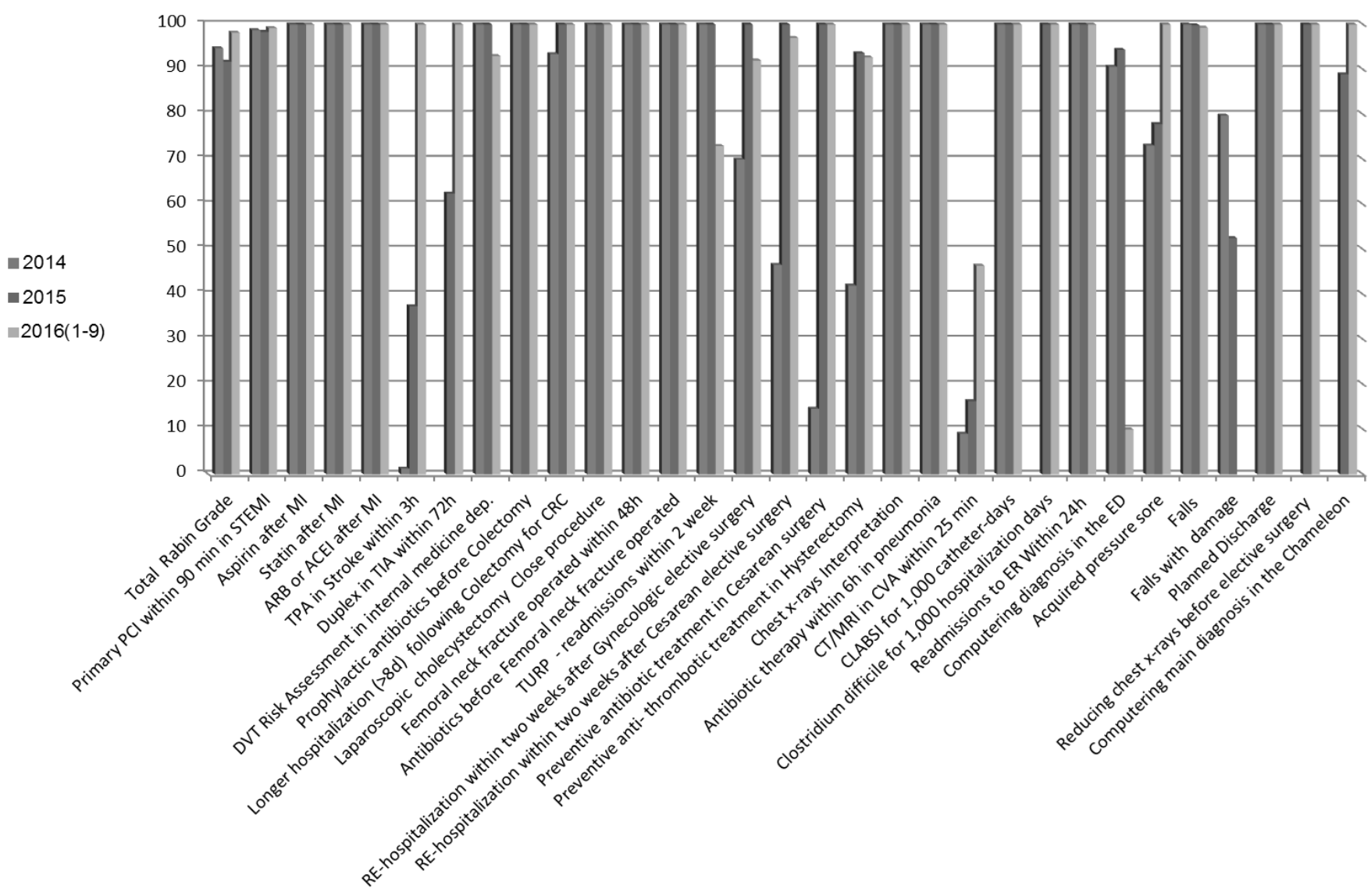

Figure 5: Core indicators measurement 2014-2016.

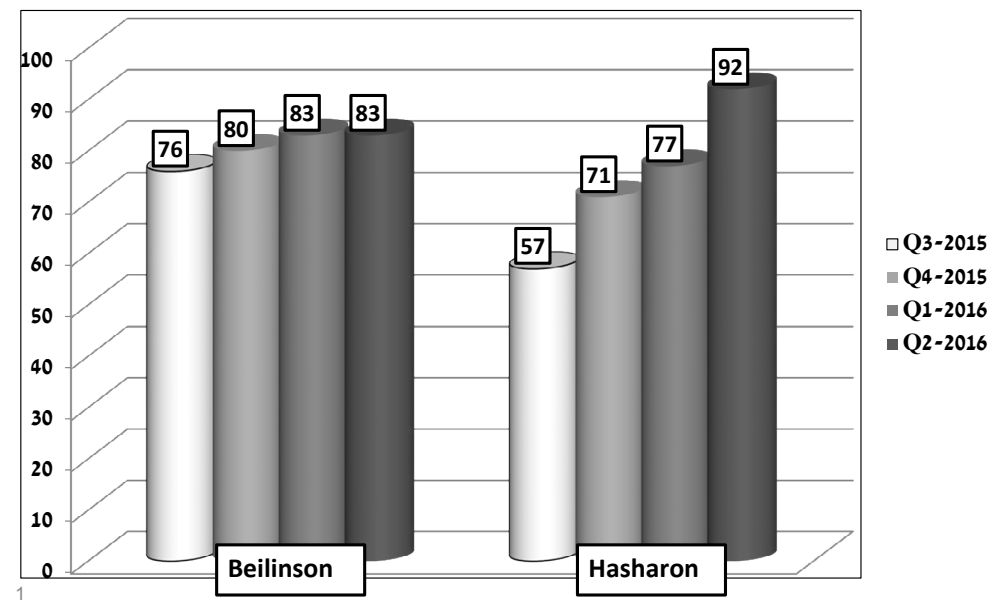

Figure 6: PCI within 90 min to STEMI (ST elevation myocardial infarction) in the emergency room.

prepared, presented to the staff, and followed closely for performance. Continuous improvement was demonstrated for each of the clinical protocol compliance and outcomes. As example is the performing PCI for STEMI patients (Figure 6).

\section{Data Collection and Validation}

Validation is performed when a new measure is implemented, a change has been made (data, source and topic) or data is made public. The responsibility for executing the validation is on the Information Technology Division, Chief Physician's office of CHS, when CHS measures are involved, the Ministry of Health for National indicators, and on the RMC Quality unit for measures developed by the hospital. Selection of sample for validation - $5 \%$ of all cases relevant to the measure during the quarter is sampled randomly (Table 1). 


\begin{tabular}{|c|c|}
\hline $\begin{array}{c}\text { Total quantity of data } \\
\text { (Size of original sample) }\end{array}$ & Sample required for validation \\
\hline $1-8$ & All data \\
\hline $9-180$ & At least 9 \\
\hline $181-1,000$ & $5 \%$ \\
\hline$>1,000$ & 50 \\
\hline
\end{tabular}

Table 1: How to validate the data?

\section{Indicator}

References

Per cutaneous coronary catheterization (PCI) within 90 min in ST elevation myocardial infarction (STEMI)

Preventive aspirin therapy for patients with acute MI

Preventive statins therapy for patients with acute MI

Performance evaluation of risk of stroke in patients with atrial fibrillation

Evaluation of Padua score for prevention of venous thrombosis

Carotid arteries duplex within $72 \mathrm{~h}$ of admission to the emergency department due to transit ischemic attack

TPA or neuro-angiography after acute CVA

Preventive antibiotic treatment an hour before colectomy

Operation of hip fracture within $48 \mathrm{~h}$ of admission

Antibiotic prophylaxis before hip surgery

Preventive antibiotic treatment before Cesarean section

Preventive anti-thrombotic treatment before hysterectomy

Steroids therapy for women at risk of preterm delivery

Hospitalization within two weeks after gynecologic elective surgery

Hospitalization within two weeks after elective cesarean section

Readmissions to the emergency department within $24 \mathrm{~h}$

Computing diagnosis in the emergency department, brain CT or MRI in acute CVA within 25 min of arrival to the emergency

Chest $\mathrm{x}$-rays interpretation

Central line associated bloodstream sepsis (CLABSI) for 1,000 catheter-days

Clostridium difficile incidence for 1,000 hospitalization days

Falls for 1000 hospitalization days

Falls with severe damage for 1000 hospitalization days

Acquired pressure sore

Computing main diagnosis in the digital patient's file

Longer hospitalization of more than 8 days following colectomy for colorectal cancer

Readmission within 2 weeks after trans urethral prostatectomy

Planned patient's discharge

Reducing chest $\mathrm{x}$-rays before elective surgery

Table 2: Quality indicators (procedures, service measurements and outcomes).

[4]

[5]

[6]

[7]

[8]

[9]

[10]

[11]

[12]

[13]

[14]

[15]

[16]

[17]

[18]

[19]

[20]

NA

[21]

[22]

[23]

[24]

[24]

NA

[25]

[26]

NA

[27]

\section{How We Prioritize Acts?}

The following parameters are important for prioritizing our acts: Significant importance, high risk, high volume, high frequency, time limitation - the challenge of narrow window of opportunities, evidence based association with significant outcome, national focus (Ministry of Health demands) and potential intervention.

\section{Internet Portal}

An internet portal has been created specifically for the QSS as an additional independent tool for use by the hospital staff members. There are sections for notifications, forms, presentations, articles, quality control, clinical guidelines and interactions between specialist clinic advisors and their primary care clinics (this was also used for the quality improvement assessment of referrals and answers to questions).

\section{Quality Indicators}

In order to assess clinical quality and define whether our efforts were appreciated and adopted by the clinical staffs, we used quality indicators, assessing improvement. Delphi process with plenary sessions and discussion groups helped to define the quality indicators which included procedures, service measurements and outcomes (Figure 6) [2,3]. We measured 28 indicators (Table 2) [4-27].

\section{Quality Improvement Plan}

Annual quality improvement plan is prepared every year, including all of the above mentioned issues, approved by the hospital management. This plan is performed according to the approved protocols and measured by the quality indicators. Achieving the set of indicator goals is considered success, and is measured for every unit of the medical center. In addition we added a care manager for every patient admitted to the hospital, starting at the admission.

\section{Summary}

Integrated quality assurance and patient's safety service was established on 2012 in Rabin Medical Center. Organizational changes focused on patient safety, based on PDCA clinical protocols, quality indicators and special committees brought the medical center to new, high level, achievements. We believe that our patients enjoyed high level quality of care in the hospital safe environment. 
Citation: Niv Y, Berkov E, Chorev N, Cohen S, Kanter P, et al. (2017) Establishing an Integrated Quality Assurance and Patient's Safety Service in a Tertially, Academic Medical Center. Health Care Current Reviews 5: 198. doi: 10.4172/2375-4273.1000198

\section{Acknowledgement}

We thank Eyran Halpern, Riki Zatland, Erez Levi, Eran Rotman, Gadi Neumann, Hagit Hendel, Efrat Milner, Cheli Levi-Sahar, Eythan Chaver, Inon Buda, Boaz Tadmor, Lara Keselman, Arnon Viznizer, Riki Gilad, Michal Liphschitz, Irit Weisbord, Iris Badnani, Natali Lanzman, members of Rabin Medical Center Management for their help and support.

\section{References}

1. www.nationalacademies.org/hmd/ /media/Files/Report\%20Files/1999/To-Erris-Human/To\%20Err\%20is\%20Human\%201999\%20\%20report\%20brief.pdf

2. Cohen A, Dreiher J, Regev- Rosenberg S, Yakovson O, Lieberman N, et al. (2010) A quality control program for Clalit Health Services: the first decade. Harefuah149: 1-6.

3. Chassin M, Loeb JM, Schmaltz SP, Wachter RM (2010) Accountability measures-using measurement to promote quality improvement. N Engl J Med 383:683-688.

4. Lambert L, Brown K, Segal E (2010) Association between timeliness of reperfusion therapy and clinical outcomes in ST-elevation myocardial infarction. JAMA 303: 2148-2155

5. APT Statistical Secretariat (1994) Collaborative overview of randomised trials of antiplatelet therapy--I: Prevention of death, myocardial infarction and stroke by prolonged antiplatelet therapy in various categories of patients. BMJ 308 : 81-106.

6. Smith SC, Blair SN, Bonow RO (2001) AHA/ACC guidelines for preventing heart attack and death in patients with atherosclerotic cardiovascular disease: 2001 update. Circulation 204: 1577-1579.

7. Hart RG, Benavente O, McBride R, Pearce LA (1999) Antithrombotic therapy to prevent stroke in patients with atrial fibrillation: A meta-analysis. Ann Intern Med 131: 492-501.

8. Roth-Yelinek, B (2012) Venous thromboembolism prophylaxis of acutely ill hospitalized medical patients. Are we under-treating our patients? Eur J Intern Med 23: 236-239.

9. Long A (2002) Critical review of non or minimally invasive methods (duplex ultrasonography, MR- and CT-angiography) for evaluating stenosis of the proximal internal carotid artery. Eur J Vas Endo Surg 24: 43-52.

10. Meyers PM, Schumacher HC, Alexander MJ, Derdeyn CP, Furlan AJ, et al. (2010) Performance and training standards for endovascular ischemic stroke treatment. J Neurosurg 113: 149-152.

11. Eskicioglu C (2010) Preoperative bowel preparation for patients undergoing elective colorectal surgery: A clinical practice guideline endorsed by the Canadian Society of Colon and Rectal Surgeons. Can J Surg 53:385-395.

12. Rae HC, Harris IA, McEvoy L, Todorova T (2007) Delay to surgery and mortality after hip fracture. ANZ J Surg 77: 889-891.
13. Bratzler D, Houck PM (2004) Antimicrobial prophylaxis for surgery and advisory statement from the National Surgical Infection Prevention Project. Clin Infect Dis 38:1706-1714.

14. Raghunathan K, Connelly NR, Friderici J (2013) Unwarranted variability in antibiotic prophylaxis for cesarean section delivery: A National Survey of Anesthesiologists. Anesthesia and Analgesia 116: 644-648.

15. Heit JA, Silverstein MD, Mohr DN (2000) Risk factors for deep vein thrombosis and pulmonary embolism: A population-based case-control study. Arch Intern Med 160: 809-815

16. Melamed N (2015) Association between antenatal corticosteroid administrationto-birth interval and outcomes of preterm neonates. Obst Gyn 125: 1377-1384.

17. Andersen TF, Loft A, Brønnum-Hansen H, Roepstorff C, Madsen M (1993) Complications after hysterectomy. A Danish population based study 19781983. Acta Obstet Gynecol Scand 72: 570-577.

18. Olsen MA, Butler AM, Willers DM, Devkota P, Gross GA et al. (2008) Risk factors for surgical site infection after low transverse cesarean section. Infect Control Hosp Epidemiol 29: 477-484.

19. Sauvin G, Freund Y, Saïdi K, Riou B, Hausfater P (2013) Unscheduled return visits to the emergency department: consequences for triage. Acad Emerg Med 20: $33-39$

20. Stroke-American Heart Association/American Stroke Association (AHA ASA) guideline recommendations for the management of patients with acute ischemic stroke. Statement from the American Heart Association. Stroke 40: 2911-2944.

21. Marschall J, Mermel LA, Classen D, Arias KM, Podgorny K, et al. (2008) Strategies to prevent central line-associated bloodstream infections in acute care hospitals. Infect Control Hosp Epidemiol 29 Suppl 1: S22-30.

22. Kelly CP, LaMont JT (2008) Clostridium difficile--more difficult than ever. N Eng J Med 359: 1932-1940.

23. Joint Commission International Standards for hospitals.

24. Moriarty JP, Finnie DM, Johnson MG, Huddleston JM, Naessens JM (2010) Do pre-existing complications affect the failure to rescue quality measures? Qual Saf Health Care 19: 65-68.

25. www.achs.org.au/pdf/Australasian_clinical indicator_Report 200108 WEB. pdf

26. Archer C, Levy AR, McGregor M (1993) Value of routine preoperative chest x-rays: A meta-analysis. Can J Anaesth 40: 1022-1027.

27. Ciccone MM, Aquilino A, Cortese F, Scicchitano P, Sassara M, et al. (2010) Feasibility and effectiveness of a disease and care management model in the primary health care system for patients with heart failure and diabetes (Project Leonardo). Vasc Health Risk Manag 6: 297-305. 